\title{
Association between serum haptoglobin and carotid arterial functions: usefulness of a targeted metabolomics approach
}

\author{
Shiyun Wang ${ }^{1 \dagger}$, Jie Wang ${ }^{1 \dagger}$, Rong Zhang ${ }^{1}$, Aihua Zhao ${ }^{2}$, Xiaojiao Zheng ${ }^{2}$, Dandan Yan ${ }^{1}$, Feng Jiang ${ }^{1}$, Wei Jia², \\ Cheng $\mathrm{Hu}^{1,3^{*}}$ and Weiping Jia ${ }^{1 *}$ (I)
}

\begin{abstract}
Background: Serum haptoglobin (Hp) has been closely associated with cardio-cerebrovascular diseases. We investigated a metabolic profile associated with circulating $\mathrm{Hp}$ and carotid arterial functions via a targeted metabolomics approach to provide insight into potential mechanisms.

Methods: A total of 240 participants, including 120 patients with type 2 diabetes mellitus (T2DM) and 120 nondiabetes mellitus (non-DM) subjects were recruited in this study. Targeted metabolic profiles of serum metabolites were determined using an AbsoluteIDQ ${ }^{\mathrm{TM}}$ p180 Kit (BIOCRATES Life Sciences AG, Innsbruck, Austria). Ultrasound of the bilateral common carotid artery was used to measure intima-media thickness and inter-adventitial diameter. Serum Hp levels were tested by enzyme-linked immunosorbent assay.

Results: Serum Hp levels in T2DM patients and non-DM subjects were $103.40(72.46,131.99) \mathrm{mg} / \mathrm{dL}$ and 100.20 $(53.99,140.66) \mathrm{mg} / \mathrm{dL}$, respectively. Significant differences of 19 metabolites and 17 metabolites were found among serum $\mathrm{Hp}$ tertiles in T2DM patients and non-DM subjects, respectively $(P<0.05)$. Of these, phosphatidylcholine acylalkyl C32:2 (PC ae C32:2) was the common metabolite observed in two populations, which was associated with the serum $\mathrm{Hp}$ groups and lipid traits $(P<0.05)$. Furthermore, the metabolite ratios of two acidic amino acids, including aspartate to PC ae C32:2 (Asp/PC ae C32:2) and glutamate to PC ae C32:2 (Glu/PC ae C32:2) were correlated with serum $\mathrm{Hp}$, carotid arterial functions and other biochemical index in both populations significantly $(P<0.05)$.
\end{abstract}

Conclusions: Targeted metabolomics analyses might provide a new insight into the potential mechanisms underlying the association between serum $\mathrm{Hp}$ and carotid arterial functions.

Keywords: Metabolites, Haptoglobin, Carotid inter-adventitial diameter, Carotid intima-media thickness, Type 2 diabetes, Non-diabetes mellitus

\section{Background}

Type 2 diabetes mellitus (T2DM) represents a heterogeneous group of chronic metabolic disorders, and its increasing prevalence has led to an explosion of the global disease burden with increased mortality and

\footnotetext{
*Correspondence: alfredhc@sjtu.edu.cn; wpjia@sjtu.edu.cn

${ }^{\dagger}$ Shiyun Wang and Jie Wang contributed equally to this work

'Shanghai Diabetes Institute, Shanghai Key Laboratory of Diabetes

Mellitus, Shanghai Clinical Center for Diabetes, Shanghai Jiao

Tong University Affiliated Sixth People's Hospital, 600 Yishan Road,

Shanghai 200233, People's Republic of China

Full list of author information is available at the end of the article
}

morbidity [1, 2]. Systemic metabolic dysfunctions, such as hyperglycaemia, insulin resistance and dyslipidaemia, give rise to an elevated risk of cardio-cerebrovascular diseases, including cerebral infarction, stroke, and heart attacks $[3,4]$. The pathogenesis underlying macroangiopathy is complicated and yet not well clarified [5-8]. A group of studies was previously launched to explore the causality underlying the relationship of several novel biomarkers with macroangiopathy risk [9-11], and a causal relationship between serum haptoglobin ( $\mathrm{Hp}$ ) levels and macroangiopathy was observed in Chinese T2DM patients via Mendelian randomization analysis [12]. 
As a kind of acute-phase response protein, serum $\mathrm{Hp}$ is mainly synthesized by hepatocytes and is widely distributed in the circulation in humans [13]. The main physiological function of serum $\mathrm{Hp}$ is binding free haemoglobin released from red blood cell lysis and protecting tissues and vessels from oxidative damage [14]. Several studies have reported that increases in circulating $\mathrm{Hp}$ levels were observed in T2DM patients and general population with cardio-cerebrovascular diseases [15-17]. Furthermore, it has been proposed that the influence of $\mathrm{Hp}$ on the cholesterol level might be related to the ability of $\mathrm{Hp}$ to bind apolipoprotein E, which is closely related to lipid metabolism and carotid atherosclerosis [18]. Thus, understanding the mechanisms underlying the interactions involving serum Hp, lipid metabolism and carotid arterial functions might provide a novel clue for the prevention and treatment of cardio-cerebrovascular diseases in T2DM patients and general population.

Recently, metabolomics has been used as a more expeditious and sensitive approach to explore changes in metabolic profiles of chronic metabolic diseases [19, 20]. Metabolites indicate intermediates and end products of metabolic pathways, including carbohydrates, fatty acids, amino acids, pigments, nucleotides, organic acids, vitamins and many other classes of compounds [21]. Changes in metabolites in organisms, tissues or cells are direct indicators of variation in the physiology and pathology of diseases [22]. In current metabolomics research, the three different approaches adopted in the measurement of metabolites are metabolic fingerprinting, metabolite profiling and targeted metabolomics [23].

In the current study, we aimed to investigate whether a targeted metabolomics approach involving a broader spectrum of metabolites may help to identify metabolites associated with the serum $\mathrm{Hp}$ levels in Chinese T2DM patients and non-diabetes mellitus (non-DM) subjects. Furthermore, analyses of the altered metabolites and metabolite ratios with the traits of carotid arterial functions, including intima-media thickness (IMT) and inter-adventitial diameter (IAD) of the bilateral common carotid arteries, were performed to provide further insight into the potential mechanisms involved.

\section{Methods}

\section{Subjects}

A total of 240 participants were enrolled in this study, including 120 T2DM patients from the Shanghai Diabetes Institute Inpatient Database and 120 non-DM subjects from the Shanghai Nicheng Cohort Study [24]. T2DM and non-DM were diagnosed by the 1999 WHO criteria following a $75 \mathrm{~g}$ oral glucose tolerance test. Written informed consent was obtained from all patients. This current study complied with the Declaration of
Helsinki and was approved by the Institutional Review Board of Shanghai Jiao Tong University Affiliated Sixth People's Hospital, Shanghai, China. Exclusion criteria were subjects with cancer, severe disability, psychiatric disturbances, type 1 diabetes, pregnancy, haemolytic disease, cardiac failure, severe infection, and drug or alcohol addiction. Fasting venous blood samples of all patients were collected and centrifuged, and the separated serum samples were transferred and stored at $-80{ }^{\circ} \mathrm{C}$ until assayed.

\section{Clinical examination}

Anthropometric characteristics of all 240 participants, such as age, sex, height, weight, and duration of diabetes (years), were recorded. Body mass index (BMI) was defined as weight $(\mathrm{kg}) /$ height $^{2}\left(\mathrm{~m}^{2}\right)$. Blood pressure was measured using a mercury sphygmomanometer by an experienced physician, and the mean values of three repeated measurements were recorded. A vascular ultrasound was used to examine the bilateral common carotid arteries. Information on the IMT and IAD was recorded in T2DM patients and IMT was used in non-DM subjects. The mean values of IMT and IAD of the bilateral common carotid arteries were calculated for further analysis. Haemoglobin A1c (HbA1c) levels were measured by a Bio-Rad Variant II haemoglobin testing system (Bio-Rad Laboratories, Hercules, USA). Blood lipid profiles, which included levels of total cholesterol, triglycerides, high-density lipoprotein-cholesterol (HDL-C), and low-density lipoprotein-cholesterol (LDL-C), were tested by a Hitachi 7600-020 Automated Analyzer (Hitachi, Tokyo, Japan).

\section{Metabolite measurements}

The metabolite measurements were conducted via a targeted metabolomics approach by using an AbsoluteIDQ ${ }^{\mathrm{TM}}$ p180 Kit (BIOCRATES Life Sciences AG, Innsbruck, Austria) combined with flow injection analysis and liquid chromatography-tandem mass spectrometry. A total of 184 metabolites were detected by this kit, including 40 acylcarnitines (C x:y), 87 glycerophospholipids [14 lysophosphatidylcholines (lyso-PCs) (lyso-PC x:y) and 73 phosphatidylcholines (PCs) (35 PC aa x:y and 38 PC ae $\mathrm{x}: \mathrm{y})], 14$ sphingolipids (SM x:y or SM (OH) x:y), 21 amino acids, 21 biogenic amines and 1 hexose. Glycerophospholipids were differentiated according to the presence of ester and ether bonds in the glycerol moiety. Double letters of "aa" or "ae" indicate that two glycerol positions are bound to a fatty acid residue via an ester bond or ether bond, whereas a single letter of "a" or "e" represents only one fatty acid residue bound to the glycerol backbone. The abbreviation " $\mathrm{C} \mathrm{x}: \mathrm{y}$ " is used to indicate the composition of a lipid fatty side chain, where " $x$ " represents the 
number of carbon atoms and "y" indicates the number of double bonds in the side chain. The assay procedures for measurements and quality control followed the kit manufacturer's instructions [25]. The samples were analysed using a Waters $\mathrm{XEVO}^{\mathrm{TM}} \mathrm{TQ}$ mass spectrometer (Waters, Manchester, UK) coupled with a Waters ACQUITY $\left({ }^{\circledR}\right)$ ultra performance liquid chromatography (UPLC). Biocrates MetIQ ${ }^{\mathrm{TM}}$ software (BIOCRATES Life Sciences AG, Innsbruck, Austria) was used to quantify metabolite concentrations and assess the quality of metabolites automatically. Identification and calculations of the metabolite concentration were achieved using internal standards. Metabolite concentrations were reported in the unit of $\mu \mathrm{mol} / \mathrm{L}(\mu \mathrm{M})$, and an Excel file was exported for further statistical analyses. Metabolites below the limit of detection were excluded from further analysis.

\section{Circulating Hp detection}

An enzyme-linked immunosorbent assay (ELISA) was used to measure Hp concentrations by using a Human Haptoglobin Quantikine ELISA kit (R\&D Systems, Inc., Minneapolis, USA) following the kit manufacturer's instructions as described previously [12].

\section{Statistical analyses}

Statistical analyses of the quantitative characteristics were carried out using SAS for Windows (version 9.2; SAS Institute, Cary, NC, USA). Variables were subjected to normality tests, and any skewed quantitative trait data were logarithmically transformed before analysis. Statistical significance of clinical traits and metabolites among three groups of serum Hp levels tertiles (low Hp, middle $\mathrm{Hp}$ and high $\mathrm{Hp}$ ) was determined by one-way analysis of variance (ANOVA) followed by Dunnett's test to identify group differences at $P<0.05$. Pearson correlation analysis was performed to evaluate correlations between two variables. Multiple linear regression analysis was used to test the association of metabolites and metabolite ratios with lipid traits after adjusting for confounding factors. All data are shown as $\mathrm{n}$, the mean \pm standard deviation or median (interquartile range). A two-tailed $P$ value $<0.05$ was considered nominally significant. A total of 184 metabolites were measured, and seven were excluded because of low detection quality. Bonferroni correction was applied to adjust for multiple testing (i.e., 177 tests for the analysis of metabolites association). Thus, associations of $P<$ adjust $P(0.05 / 177)=2.8 \times 10^{-4}$ for metabolites and serum $\mathrm{Hp}$ were considered significant. Comprehensive meta-analysis (version 2.2.057; Biostat, Englewood, New Jersey) was conducted to evaluate combined effects from two populations using a fixed or random effect model after testing for heterogeneity. The
Cochran $Q$ statistic and the $I^{2}$ statistic were applied to assess the extent of heterogeneity.

\section{Results \\ Basic clinical features}

The median (interquartile range) values of serum $\mathrm{Hp} \mathrm{lev-}$ els in T2DM patients and non-DM subjects were 103.40 $(72.46,131.99) \mathrm{mg} / \mathrm{dL}$ and $100.20(53.99,140.66) \mathrm{mg} / \mathrm{dL}$, respectively. No significant difference was found in serum Hp levels between these two populations $(P=0.7315)$. Anthropometric and clinical characteristics of all the 240 participants grouped by serum $\mathrm{Hp}$ level tertiles are described in Table 1.

In T2DM patients, the median (interquartile range) values of serum $\mathrm{Hp}$ concentrations in low $\mathrm{Hp}$, middle $\mathrm{Hp}$ and high Hp groups were $54.82(3.60,71.58) \mathrm{mg} / \mathrm{dL}$, 103.20 (95.30, 107.27) $\mathrm{mg} / \mathrm{dL}$ and 151.64 (131.12, 175.55) $\mathrm{mg} / \mathrm{dL}$, respectively. After comparison among groups, significant differences were found in sex $(P=0.0410)$, carotid IAD $(P=0.0088)$ and serum Hp levels $(P<0.0001)$ (Fig. 1). Furthermore, after adjusting for age, sex, BMI, blood pressure, duration of diabetes and HbA1c levels, the carotid IAD parameter was shown to have a significant relationship with serum Hp tertiles $(P=0.0042)$ (see Additional file 1: Table S1).

In non-DM subjects, the median (interquartile range) values of serum $\mathrm{Hp}$ concentrations in low $\mathrm{Hp}$, middle Hp and high Hp groups were 39.48 (17.23, 51.45) mg/ $\mathrm{dL}, 99.41$ (92.96, 113.38) $\mathrm{mg} / \mathrm{dL}$ and 167.67 (140.35, 209.73) $\mathrm{mg} / \mathrm{dL}$, respectively, with a significant difference of serum $\mathrm{Hp}$ levels among three groups $(P<0.0001)$ (see Additional file 1: Figure S1). After adjusting for age, sex, BMI, blood pressure and HbA1c levels, no parameters was shown to have a significant relationship with serum Hp tertiles $(P>0.05)$ (see Additional file 1: Table S2).

\section{Association between blood metabolites and serum $\mathrm{Hp}$ levels}

A total of 184 metabolites were measured, and seven were excluded because of low detection quality. As a result, significant differences of 19 metabolites (2 acylcarnitines, 1 lyso-PCs, 12 PCs, and 4 amino acids) and 17 metabolites (5 acylcarnitines, 2 lyso-PCs, 7 PCs, and 3 amino acids) were found among the $\mathrm{Hp}$ level groups from the remaining 177 metabolites in T2DM patients and non-DM subjects, respectively $(P<0.05)$ (Table 2$)$. After further linear regression analysis, 41 metabolites (1 acylcarnitine, 3 lyso-PCs, 33 PCs and 4 amino acids) and 17 metabolites ( 3 acylcarnitine, 1 lyso-PCs, 10 PCs and 3 amino acids) were found to be associated with serum $\mathrm{Hp}$ levels $(P<0.05)$ in T2DM patients and non-DM subjects, respectively (see Additional file 1: Table S3). 
Table 1 Basic clinical characteristics of subjects in this study

\begin{tabular}{|c|c|c|c|c|}
\hline Variable & Low Hp & Middle Hp & High Hp & $P$ value \\
\hline \multicolumn{5}{|l|}{ T2DM $(n=120)$} \\
\hline Number & 39 & 40 & 41 & - \\
\hline Age (years) & $56.85 \pm 11.18$ & $56.88 \pm 12.16$ & $58.25 \pm 11.46$ & 0.8245 \\
\hline Male/female & $30 / 9$ & $22 / 18$ & $32 / 9$ & 0.0410 \\
\hline $\mathrm{BMI}\left(\mathrm{kg} / \mathrm{m}^{2}\right)$ & $24.69 \pm 4.24$ & $24.63 \pm 3.25$ & $24.62 \pm 2.89$ & 0.9959 \\
\hline $\mathrm{SBP}(\mathrm{mmHg})$ & $130(120,150)$ & $127(110,134)$ & $130(120,150)$ & 0.0852 \\
\hline $\mathrm{DBP}(\mathrm{mmHg})$ & $80(74,90)$ & $75(70,82)$ & $80(72,90)$ & 0.1756 \\
\hline Duration of diabetes (years) & $10(4,15)$ & $10(7,10)$ & $10(4,15)$ & 0.8267 \\
\hline $\mathrm{HbA1c}(\%)$ & $8.00(7.20,10.20)$ & $8.40(7.20,10.30)$ & $8.30(7.20,9.70)$ & 0.9903 \\
\hline Total cholesterol (mmol/L) & $4.64(4.28,5.59)$ & $4.61(3.78,5.37)$ & $4.40(3.87,4.87)$ & 0.3833 \\
\hline Triglycerides (mmol/L) & $1.50(1.20,2.20)$ & $1.29(0.79,1.93)$ & $1.39(1.03,1.65)$ & 0.2756 \\
\hline $\mathrm{HDL}-\mathrm{C}(\mathrm{mmol} / \mathrm{L})$ & $1.09(0.95,1.20)$ & $0.99(0.88,1.24)$ & $1.00(0.81,1.10)$ & 0.2181 \\
\hline LDL-C (mmol/L) & $2.76 \pm 0.85$ & $2.74 \pm 0.76$ & $2.65 \pm 0.84$ & 0.7488 \\
\hline Carotid IMT (mm) & $0.80(0.70,0.90)$ & $0.75(0.65,0.90)$ & $0.80(0.70,0.90)$ & 0.3466 \\
\hline Carotid IAD (mm) & $6.17 \pm 0.70$ & $6.28 \pm 0.78$ & $6.65 \pm 0.67$ & 0.0088 \\
\hline Serum Hp levels (mg/dL) & $54.82(3.60,71.58)$ & $103.20(95.30,107.27)$ & $151.64(131.12,175.55)$ & $<0.0001$ \\
\hline \multicolumn{5}{|l|}{ Non-DM $(n=120)$} \\
\hline Number & 39 & 40 & 41 & - \\
\hline Age (years) & $64.06 \pm 4.31$ & $64.10 \pm 4.27$ & $64.58 \pm 4.15$ & 0.8720 \\
\hline Male/female & $17 / 22$ & $20 / 20$ & 23/18 & 0.5378 \\
\hline BMI $\left(\mathrm{kg} / \mathrm{m}^{2}\right)$ & $24.11 \pm 3.27$ & $25.09 \pm 3.18$ & $25.64 \pm 4.06$ & 0.1522 \\
\hline $\mathrm{SBP}(\mathrm{mmHg})$ & $125(120,139)$ & $129(122,138)$ & $129(123,138)$ & 0.5074 \\
\hline $\mathrm{DBP}(\mathrm{mmHg})$ & $81(78,87)$ & $81(78,83)$ & $82(80,87)$ & 0.4709 \\
\hline $\mathrm{HbA1c}(\%)$ & $5.64 \pm 0.59$ & $5.61 \pm 0.65$ & $5.82 \pm 0.51$ & 0.1398 \\
\hline Total cholesterol (mmol/L) & $4.36(4.00,4.89)$ & $4.49(3.98,4.97)$ & $4.40(4.07,4.79)$ & 0.8007 \\
\hline Triglycerides (mmol/L) & $1.03(0.80,1.43)$ & $1.10(0.89,1.41)$ & $0.99(0.80,1.26)$ & 0.7676 \\
\hline $\mathrm{HDL}-\mathrm{C}(\mathrm{mmol} / \mathrm{L})$ & $1.31(1.09,1.58)$ & $1.29(1.17,1.52)$ & $1.22(1.14,1.41)$ & 0.6175 \\
\hline LDL-C (mmol/L) & $2.53(2.25,2.89)$ & $2.56(2.15,2.97)$ & $2.61(2.28,2.91)$ & 0.7962 \\
\hline Carotid IMT (mm) & $0.64 \pm 0.10$ & $0.65 \pm 0.09$ & $0.66 \pm 0.08$ & 0.4667 \\
\hline Serum Hp levels (mg/dL) & $39.48(17.23,51.45)$ & $99.41(92.96,113.38)$ & $167.67(140.35,209.73)$ & $<0.0001$ \\
\hline
\end{tabular}

Data are shown as $\mathrm{n}$ or the mean \pm standard deviation or median (interquartile range)

T2DM type 2 diabetes mellitus, Non-DM non-diabetes mellitus, $H p$ haptoglobin, $B M I$ body mass index, $S B P$ systolic blood pressure, $D B P$ diastolic blood pressure, $H D L-C$ high-density lipoprotein-cholesterol, $L D L-C$ low-density lipoprotein-cholesterol, IMT intima-media thickness, IAD inter-adventitial diameter

$P$ values $<0.05$ are shown in italic

\section{Selected metabolites associated with blood lipid traits}

Multiple linear regression analysis was conducted to investigate the association of the altered metabolites with traits of blood lipids and carotid arterial functions (carotid IMT and carotid IAD). After adjusting for age, sex and BMI, 10 metabolites (1 lyso-PC, 7 PCs and 2 amino acids) and 10 metabolites (4 lyso-PC, 5 PCs and 1 amino acids) were found to be associated with the serum $\mathrm{Hp}$ groups and lipid traits in T2DM patients (Table 3) and non-DM subjects (Table 4), respectively. Among them, phosphatidylcholine acyl-alkyl C32:2 (PC ae C32:2) was the common metabolite associated with the serum Hp groups and lipid traits in both two populations. In T2DM patients, PC ae C32:2 was significantly correlated with total cholesterol $(P=0.0390)$, the serum Hp groups $(P=0.0005)$ and carotid IAD $(P=0.0182)$. In non-DM subjects, $P C$ ae $C 32: 2$ was associated with HDL-C levels $(P=0.0133)$ and the serum Hp groups $(P=0.0390)$ significantly. The combined analysis of two populations by a meta-analysis revealed that $\mathrm{PC}$ ae $\mathrm{C} 32: 2$ was correlated with the serum $\mathrm{Hp}$ groups $(\beta \pm \mathrm{SE}=-0.028 \pm 0.007$, $\left.P=3.01 \times 10^{-5}\right)$. The Cochran $Q$ test showed no heterogeneity in the relationships between PC ae C32:2 and serum Hp from T2DM patients and non-DM subjects $\left(I^{2}=0, P=0.874\right)$. The levels of PC ae C32:2 in T2DM patients and non-DM subjects grouped by serum Hp levels are shown in Fig. 2. 

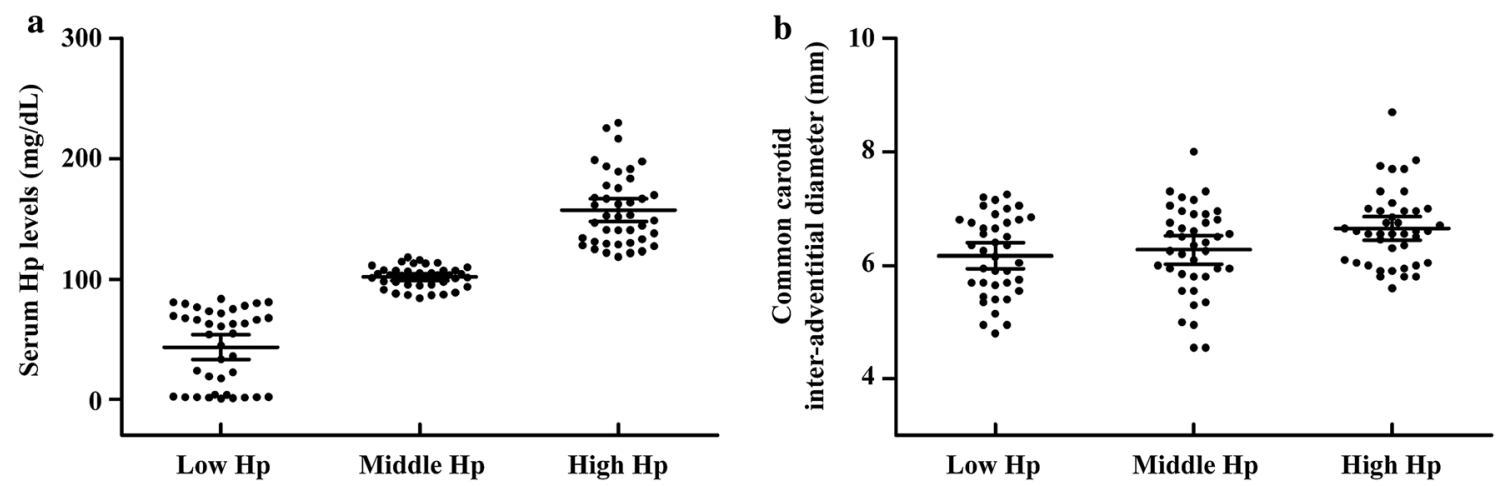

Fig. 1 Serum haptoglobin levels and common carotid inter-adventitial diameter in T2DM patients. T2DM type 2 diabetes mellitus, Hp haptoglobin. Dot plot a shows a comparison of serum $\mathrm{Hp}$ levels among Hp tertiles, $P<0.0001$. Dot plot $\mathbf{b}$ shows a comparison of common carotid artery inter-adventitial diameter levels among $\mathrm{Hp}$ tertiles, $P=0.0090$. $P$ values were determined by one-way analysis of variance. The $\mathrm{Hp}$ and carotid artery inter-adventitial diameter levels are shown in dot plots; the mean is indicated by the middle black solid line. The $95 \%$ confidence intervals are shown by the bottom and top black solid lines, respectively

\section{Metabolite ratios associated with clinical traits}

In addition, changes in metabolite ratios might reflect biological situations such as changes in enzyme activities or imbalances in metabolic pathways [26]. The metabolite ratio of valine to $\mathrm{PC}$ ae $\mathrm{C} 32: 2$ ( Val/PC ae C32:2) was previously reported to be associated with an increased risk of type 2 diabetes and measures of insulin secretion and resistance [27]. In the current study, the ratios of 21 amino acids to $\mathrm{PC}$ ae C32:2 were compared to evaluate the association between serum $\mathrm{Hp}$ levels and clinical traits.

We found the metabolite ratios of 5 aliphatic amino acids (asparagine, aspartate, glutamate, isoleucine and valine) to $\mathrm{PC}$ ae C32:2 were associated with blood lipids, the serum Hp groups, and carotid IMT and/or IAD after adjusting for age, sex and BMI in T2DM patients $(P<0.05)$ (Table 5 and Additional file 1: Table S4). In nonDM subjects, the metabolite ratios of three amino acids (aspartate, glutamate, and phenylalanine) to PC ae C32:2 were associated with blood lipids, the serum Hp groups and carotid IMT after adjusting for age, sex and BMI $(P<0.05)$ (Table 6 and Additional file 1: Table S5).

Of these, the metabolite ratios of two acidic amino acids, including aspartate to PC ae C32:2 (Asp/PC ae C32:2) and glutamate to PC ae C32:2 (Glu/PC ae C32:2) were the common significant ratios observed in both populations. The combined analysis of two populations by a meta-analysis revealed that Asp/PC ae C32:2 and Glu/PC ae C32:2 were correlated with the serum $\mathrm{Hp}$ groups $\left(\beta \pm \mathrm{SE}=0.045 \pm 0.010, \quad P=1.45 \times 10^{-5}\right.$; $\left.\beta \pm \mathrm{SE}=0.041 \pm 0.011, \quad P=2.38 \times 10^{-4}\right), \quad$ respectively. The Cochran $Q$ test showed no heterogeneity in the relationships of Asp/PC ae C32:2 and Glu/PC ae C32:2 with serum $\mathrm{Hp}$ from T2DM patients and non-DM subjects
$\left(I^{2}=0, \quad P=0.740 ; \quad I^{2}=0, \quad P=0.860\right)$, respectively. The ratios of Asp/PC ae C32:2 and Glu/PC ae C32:2 in T2DM patients and non-DM subjects grouped by serum $\mathrm{Hp} \mathrm{lev-}$ els are shown in Figs. 3, 4, respectively.

\section{Discussion}

In the current study, serum Hp levels in T2DM patients and non-DM subjects were $103.40(72.46,131.99) \mathrm{mg} /$ $\mathrm{dL}$ and $100.20(53.99,140.66) \mathrm{mg} / \mathrm{dL}$, respectively. Significant differences of 19 metabolites and 17 metabolites were found among serum $\mathrm{Hp}$ tertiles in T2DM patients and non-DM subjects, respectively. Among them, the phosphatidylcholine PC ae C32:2 was shown to be notably correlated with the serum $\mathrm{Hp}$ groups and lipid traits in both two populations. Furthermore, the metabolite ratios of two acidic amino acids, including aspartate to $\mathrm{PC}$ ae C32:2 (Asp/PC ae C32:2) and glutamate to PC ae C32:2 (Glu/PC ae C32:2) were associated with serum Hp, carotid arterial functions and other biochemical index significantly. These findings might provide a new insight into the potential mechanisms underlying the association between serum Hp and carotid arterial functions.

An acute-phase glycoprotein, Hp was identified in 1938 for the first time. This protein is produced mostly by hepatocytes and is widely distributed in the circulation in humans. The main physiological function of $\mathrm{Hp}$ is binding free haemoglobin with high affinity to form a stable haemoglobin-haptoglobin ( $\mathrm{Hb}-\mathrm{Hp}$ ) complex, thereby protecting tissues and vessels from oxidative damage. In the range of $50-300 \mathrm{mg} / \mathrm{dL}$, the circulating level of Hp ranks only behind the predominant plasma proteins albumin and immunoglobulins [13]. Several studies reported that circulating Hp levels were correlated with the progression of cardio-cerebrovascular diseases in 
Table 2 Blood metabolites in subjects grouped by serum Hp levels

\begin{tabular}{|c|c|c|c|c|}
\hline Metabolites & Low Hp & Middle Hp & High Hp & $P$ value \\
\hline \multicolumn{5}{|l|}{$\operatorname{T2DM}(n=120)$} \\
\hline \multicolumn{5}{|l|}{ Acylcarnitines $(\mu \mathrm{M})$} \\
\hline C12:1 & $0.142 \pm 0.042$ & $0.148 \pm 0.044$ & $0.116 \pm 0.032$ & 0.0017 \\
\hline C14:1 & $0.241 \pm 0.064$ & $0.229 \pm 0.067$ & $0.201 \pm 0.048$ & 0.0119 \\
\hline \multicolumn{5}{|c|}{ Lyso-phosphatidylcholines ( $\mu \mathrm{M})$} \\
\hline Lyso-PC a C20:3 & $1.284 \pm 0.423$ & $1.050 \pm 0.319$ & $1.064 \pm 0.332$ & 0.0158 \\
\hline \multicolumn{5}{|c|}{ Phosphatidylcholines ( $\mu \mathrm{M})$} \\
\hline PC aa C32:1 & $5.437 \pm 2.670$ & $4.678 \pm 1.994$ & $4.128 \pm 1.775$ & 0.0398 \\
\hline PC aa C34:1 & $109.683 \pm 37.529$ & $99.877 \pm 34.435$ & $89.110 \pm 23.902$ & 0.0117 \\
\hline PC aa C34:2 & $323.235 \pm 80.457$ & $308.206 \pm 90.611$ & $283.969 \pm 72.902$ & 0.0456 \\
\hline PC aa C36:1 & $22.898 \pm 6.344$ & $21.283 \pm 4.553$ & $19.615 \pm 6.216$ & 0.0041 \\
\hline PC aa C40:4 & $2.093 \pm 0.646$ & $1.881 \pm 0.541$ & $1.809 \pm 0.594$ & 0.0458 \\
\hline PC ae C32:1 & $1.329 \pm 0.294$ & $1.281 \pm 0.268$ & $1.171 \pm 0.245$ & 0.0364 \\
\hline PC ae C32:2 & $0.335 \pm 0.088$ & $0.301 \pm 0.062$ & $0.281 \pm 0.058$ & 0.0094 \\
\hline PC ae C34:1 & $3.305 \pm 0.712$ & $3.254 \pm 0.733$ & $2.902 \pm 0.559$ & 0.0270 \\
\hline PC ae C36:0 & $0.577 \pm 0.180$ & $0.546 \pm 0.128$ & $0.470 \pm 0.098$ & 0.0055 \\
\hline PC ae C36:1 & $4.953 \pm 0.948$ & $4.642 \pm 0.925$ & $4.417 \pm 0.814$ & 0.0327 \\
\hline PC ae C40:5 & $2.140 \pm 0.418$ & $2.034 \pm 0.527$ & $1.871 \pm 0.374$ & 0.0329 \\
\hline PC ae C42:2 & $0.333 \pm 0.080$ & $0.307 \pm 0.082$ & $0.287 \pm 0.060$ & 0.0443 \\
\hline \multicolumn{5}{|l|}{ Amino acids $(\mu \mathrm{M})$} \\
\hline His & $115.000 \pm 15.508$ & $111.528 \pm 15.191$ & $105.444 \pm 14.510$ & 0.0183 \\
\hline Lys & $330.411 \pm 57.377$ & $306.053 \pm 61.629$ & $300.033 \pm 54.178$ & 0.0255 \\
\hline Trp & $65.594 \pm 14.239$ & $59.247 \pm 13.020$ & $58.258 \pm 10.826$ & 0.0238 \\
\hline Tyr & $89.152 \pm 19.114$ & $84.488 \pm 17.566$ & $79.436 \pm 16.558$ & 0.0494 \\
\hline \multicolumn{5}{|l|}{ Non-DM $(n=120)$} \\
\hline \multicolumn{5}{|l|}{ Acylcarnitines $(\mu \mathrm{M})$} \\
\hline $\mathrm{C} 16: 1-\mathrm{OH}$ & $0.018 \pm 0.004$ & $0.010 \pm 0.002$ & $0.013 \pm 0.002$ & 0.0440 \\
\hline $\mathrm{C} 2$ & $6.327 \pm 2.720$ & $5.684 \pm 1.558$ & $6.773 \pm 2.023$ & 0.0385 \\
\hline C3 & $0.334 \pm 0.109$ & $0.299 \pm 0.124$ & $0.386 \pm 0.191$ & 0.0294 \\
\hline $\mathrm{C} 4$ & $0.211 \pm 0.075$ & $0.184 \pm 0.082$ & $0.225 \pm 0.090$ & 0.0209 \\
\hline C6/C4:1-DC & $0.085 \pm 0.038$ & $0.083 \pm 0.058$ & $0.113 \pm 0.074$ & 0.0240 \\
\hline \multicolumn{5}{|c|}{ Lyso-phosphatidylcholines ( $\mu \mathrm{M})$} \\
\hline LysoPC a C17:0 & $1.302 \pm 0.361$ & $1.208 \pm 0.318$ & $1.115 \pm 0.326$ & 0.0463 \\
\hline LysoPC a C24:0 & $0.371 \pm 0.121$ & $0.312 \pm 0.112$ & $0.307 \pm 0.120$ & 0.0450 \\
\hline \multicolumn{5}{|c|}{ Phosphatidylcholines ( $\mu \mathrm{M})$} \\
\hline PC aa C32:3 & $0.260 \pm 0.047$ & $0.239 \pm 0.061$ & $0.225 \pm 0.059$ & 0.0087 \\
\hline PC aa C42:2 & $0.276 \pm 0.062$ & $0.289 \pm 0.116$ & $0.243 \pm 0.070$ & 0.0326 \\
\hline PC ae C32:2 & $0.475 \pm 0.098$ & $0.453 \pm 0.131$ & $0.424 \pm 0.089$ & 0.0602 \\
\hline PC ae C36:2 & $7.123 \pm 1.446$ & $6.882 \pm 2.063$ & $6.309 \pm 1.124$ & 0.0304 \\
\hline PC ae C38:0 & $1.141 \pm 0.298$ & $1.062 \pm 0.377$ & $0.969 \pm 0.252$ & 0.0471 \\
\hline PC ae C40:1 & $1.286 \pm 0.246$ & $1.164 \pm 0.263$ & $1.101 \pm 0.189$ & 0.0071 \\
\hline PC ae C42:1 & $0.372 \pm 0.093$ & $0.347 \pm 0.091$ & $0.321 \pm 0.071$ & 0.0197 \\
\hline \multicolumn{5}{|l|}{ Amino acids $(\mu \mathrm{M})$} \\
\hline Asp & $32.646 \pm 5.990$ & $31.854 \pm 4.664$ & $36.137 \pm 6.343$ & 0.0034 \\
\hline Trp & $75.043 \pm 8.173$ & $71.877 \pm 9.742$ & $68.875 \pm 11.423$ & 0.0244 \\
\hline Ac-Orn & $2.189 \pm 0.159$ & $2.239 \pm 0.001$ & $1.596 \pm 0.941$ & 0.0185 \\
\hline
\end{tabular}

Data are shown as the mean \pm standard deviation. $P$ values $<0.05$ are shown in table T2DM type 2 diabetes mellitus, Non-DM non-diabetes mellitus, Hp haptoglobin, His histidine, Lys lysine, Trp tryptophan, Tyr tyrosine, Asp aspartate, Ac-Orn acylornithine 
Table 3 Blood metabolites correlated with blood lipids and serum Hp levels in T2DM patients

\begin{tabular}{|c|c|c|c|c|c|c|c|}
\hline Metabolites & Total cholesterol & Triglycerides & HDL-C & LDL-C & Serum Hp & Carotid IMT & Carotid IAD \\
\hline \multicolumn{8}{|l|}{ Acylcarnitines $(\mu \mathrm{M})$} \\
\hline \multirow[t]{2}{*}{$\mathrm{C} 16-\mathrm{OH}$} & $0.017 \pm 0.011$ & $-0.002 \pm 0.002$ & $-0.011 \pm 0.005$ & $-0.008 \pm 0.006$ & $-0.001 \pm 0.001$ & $0.012 \pm 0.004$ & $-0.002 \pm 0.007$ \\
\hline & 0.1099 & 0.4393 & 0.0270 & 0.1690 & 0.0452 & 0.0074 & 0.7911 \\
\hline \multicolumn{8}{|c|}{ Lyso-phosphatidylcholines ( $\mu \mathrm{M})$} \\
\hline \multirow[t]{2}{*}{ Lyso-PC a C20:3 } & $-0.316 \pm 1.027$ & $0.834 \pm 0.233$ & $1.073 \pm 0.485$ & $-0.202 \pm 0.580$ & $-0.092 \pm 0.041$ & $-0.173 \pm 0.404$ & $0.511 \pm 0.693$ \\
\hline & 0.7588 & 0.0005 & 0.0290 & 0.7286 & 0.0272 & 0.6698 & 0.4621 \\
\hline \multicolumn{8}{|c|}{ Phosphatidylcholines ( $\mu \mathrm{M})$} \\
\hline \multirow[t]{2}{*}{ PC aa C32:0 } & $10.436 \pm 4.062$ & $1.295 \pm 0.921$ & $1.681 \pm 1.918$ & $-2.026 \pm 2.294$ & $-0.356 \pm 0.163$ & $2.011 \pm 1.597$ & $3.939 \pm 2.741$ \\
\hline & 0.0116 & 0.1626 & 0.3828 & 0.3791 & 0.0308 & 0.2106 & 0.1536 \\
\hline \multirow[t]{2}{*}{ PC aa C32:1 } & $-4.69 \pm 5.368$ & $5.805 \pm 1.217$ & $6.355 \pm 2.534$ & $5.062 \pm 3.032$ & $-0.458 \pm 0.215$ & $-0.323 \pm 2.11$ & $2.788 \pm 3.622$ \\
\hline & 0.3843 & $<0.0001$ & 0.0137 & 0.0979 & 0.0356 & 0.8787 & 0.4432 \\
\hline \multirow[t]{2}{*}{ PC aa C34:1 } & $-11.594 \pm 75.657$ & $92.794 \pm 17.157$ & $89.522 \pm 35.716$ & $57.696 \pm 42.730$ & $-7.002 \pm 3.033$ & $-2.744 \pm 29.739$ & $34.784 \pm 51.050$ \\
\hline & 0.8785 & $<0.0001$ & 0.0137 & 0.1798 & 0.0229 & 0.9267 & 0.4971 \\
\hline \multirow[t]{2}{*}{ PC aa C34:4 } & $0.095 \pm 0.634$ & $0.617 \pm 0.144$ & $0.824 \pm 0.299$ & $0.145 \pm 0.358$ & $-0.054 \pm 0.025$ & $-0.193 \pm 0.249$ & $-0.092 \pm 0.428$ \\
\hline & 0.8806 & $<0.0001$ & 0.0070 & 0.6861 & 0.0370 & 0.4397 & 0.8303 \\
\hline \multirow[t]{2}{*}{ PC aa C36:3 } & $14.350 \pm 36.596$ & $49.046 \pm 8.299$ & $69.044 \pm 17.276$ & $-5.324 \pm 20.669$ & $-3.283 \pm 1.467$ & $-9.846 \pm 14.385$ & $10.639 \pm 24.693$ \\
\hline & 0.6957 & $<0.0001$ & 0.0001 & 0.7972 & 0.0273 & 0.4951 & 0.6674 \\
\hline \multirow[t]{2}{*}{ PC ae C32:1 } & $0.972 \pm 0.740$ & $-0.094 \pm 0.168$ & $0.437 \pm 0.349$ & $0.072 \pm 0.418$ & $-0.078 \pm 0.030$ & $-0.054 \pm 0.291$ & $0.964 \pm 0.499$ \\
\hline & 0.1918 & 0.5766 & 0.2136 & 0.8629 & 0.0095 & 0.8543 & 0.0561 \\
\hline \multirow[t]{2}{*}{ PC ae C32:2 } & $0.411 \pm 0.196$ & $-0.033 \pm 0.045$ & $0.081 \pm 0.093$ & $-0.136 \pm 0.111$ & $-0.028 \pm 0.008$ & $-0.073 \pm 0.077$ & $0.318 \pm 0.133$ \\
\hline & 0.0390 & 0.4613 & 0.3837 & 0.2241 & 0.0005 & 0.3475 & 0.0182 \\
\hline \multirow[t]{2}{*}{ PC ae C34:1 } & $2.502 \pm 1.734$ & $0.505 \pm 0.393$ & $1.905 \pm 0.819$ & $-0.554 \pm 0.979$ & $-0.171 \pm 0.070$ & $0.759 \pm 0.682$ & $1.971 \pm 1.170$ \\
\hline & 0.1519 & 0.2021 & 0.0218 & 0.5728 & 0.0155 & 0.2678 & 0.0950 \\
\hline \multirow[t]{2}{*}{ PC ae C36:5 } & $10.917 \pm 4.572$ & $-1.698 \pm 1.037$ & $2.473 \pm 2.158$ & $-2.333 \pm 2.582$ & $-0.389 \pm 0.183$ & $-2.415 \pm 1.797$ & $0.752 \pm 3.085$ \\
\hline & 0.0187 & 0.1044 & 0.2544 & 0.3683 & 0.0360 & 0.1818 & 0.8079 \\
\hline \multirow[t]{2}{*}{ PC ae C38:6 } & $5.671 \pm 3.030$ & $-0.272 \pm 0.687$ & $2.976 \pm 1.431$ & $-0.609 \pm 1.711$ & $-0.252 \pm 0.121$ & $-1.226 \pm 1.191$ & $0.707 \pm 2.045$ \\
\hline & 0.0640 & 0.6927 & 0.0399 & 0.7227 & 0.0407 & 0.3058 & 0.7303 \\
\hline \multirow[t]{2}{*}{ PC ae C40:5 } & $2.559 \pm 1.247$ & $-0.166 \pm 0.283$ & $0.694 \pm 0.589$ & $-0.934 \pm 0.704$ & $-0.111 \pm 0.050$ & $-0.314 \pm 0.490$ & $0.182 \pm 0.841$ \\
\hline & 0.0426 & 0.5596 & 0.2413 & 0.1877 & 0.0286 & 0.5231 & 0.8287 \\
\hline \multicolumn{8}{|l|}{ Amino acids $(\mu \mathrm{M})$} \\
\hline \multirow[t]{2}{*}{ His } & $-150.758 \pm 41.991$ & $37.965 \pm 9.523$ & $39.971 \pm 19.823$ & $65.304 \pm 23.716$ & $-4.080 \pm 1.683$ & $6.756 \pm 16.506$ & $-17.531 \pm 28.334$ \\
\hline & 0.0005 & 0.0001 & 0.0463 & 0.0069 & 0.0170 & 0.6831 & 0.5374 \\
\hline \multirow[t]{2}{*}{ Phe } & $-36.314 \pm 54.241$ & $13.975 \pm 12.301$ & $-7.065 \pm 25.606$ & $33.892 \pm 30.634$ & $-4.385 \pm 2.175$ & $57.330 \pm 21.321$ & $41.455 \pm 36.600$ \\
\hline & 0.5046 & 0.2584 & 0.7831 & 0.2711 & 0.0462 & 0.0083 & 0.2599 \\
\hline
\end{tabular}

Multiple linear regression analysis was used to perform the analysis after adjusting for age, sex and body mass index with total cholesterol, triglycerides, HDL-C, LDL-C, serum $\mathrm{Hp}$ level tertiles, carotid IMT and IAD as independent variables. $\beta \pm$ SE and $P$ values are shown in table

T2DM type 2 diabetes mellitus, $H p$ haptoglobin, His histidine, Phe phenylalanine, $H D L-C$ high-density lipoprotein-cholesterol, $L D L-C$ low-density lipoproteincholesterol, IMT intima-media thickness, IAD inter-adventitial diameter

$P$ values $<0.05$ are shown in italic

T2DM patients and general population [16, 17]. Furthermore, a causal relationship between serum Hp levels and macroangiopathy was previously observed in Chinese T2DM patients via Mendelian randomization analysis [12]. Therefore, it is important to study the mechanisms underlying the interactions involving serum $\mathrm{Hp}$ and carotid arterial functions to provide a novel insight into the prevention and treatment for cardio-cerebrovascular diseases.
The advent of metabonomics, a new rapidly developing technology, offers a more powerful and sensitive means to explain the aetiology of complex chronic diseases [19, 22]. Glycerophospholipids including PCs and lyso-PCs have recently gained attention as potential biomarkers of cardiovascular disease [28, 29]. PCs not only play a key role as a component of cellular membranes but also act as a reservoir for fatty acids [30]. Lyso-PCs mostly come from the partial hydrolysis of 
Table 4 Blood metabolites correlated with blood lipids and serum Hp levels in non-DM subjects

\begin{tabular}{|c|c|c|c|c|c|c|}
\hline Metabolites & Total cholesterol & Triglycerides & HDL-C & LDL-C & Serum Hp & Carotid IMT \\
\hline \multicolumn{7}{|c|}{ Lyso-phosphatidylcholines ( $\mu \mathrm{M})$} \\
\hline \multirow[t]{2}{*}{ LysoPC a C16:0 } & $-494.124 \pm 89.651$ & $39.362 \pm 16.748$ & $133.887 \pm 36.720$ & $310.854 \pm 52.472$ & $-5.264 \pm 2.417$ & $25.137 \pm 34.301$ \\
\hline & $<0.0001$ & 0.0207 & 0.0004 & $<0.0001$ & 0.0317 & 0.4653 \\
\hline \multirow[t]{2}{*}{ LysoPC a C17:0 } & $-3.953 \pm 1.356$ & $0.401 \pm 0.253$ & $1.140 \pm 0.555$ & $2.706 \pm 0.793$ & $-0.088 \pm 0.037$ & $-0.303 \pm 0.519$ \\
\hline & 0.0043 & 0.1160 & 0.0425 & 0.0009 & 0.0183 & 0.5606 \\
\hline \multirow[t]{2}{*}{ LysoPC a C18:2 } & $52.100 \pm 26.226$ & $-2.782 \pm 4.899$ & $-0.547 \pm 10.742$ & $-28.897 \pm 15.350$ & $-1.675 \pm 0.707$ & $-5.400 \pm 10.034$ \\
\hline & 0.0496 & 0.5714 & 0.9595 & 0.0626 & 0.0197 & 0.5916 \\
\hline \multirow[t]{2}{*}{ LysoPC a C24:0 } & $-1.237 \pm 0.515$ & $0.239 \pm 0.096$ & $0.724 \pm 0.211$ & $0.648 \pm 0.301$ & $-0.031 \pm 0.014$ & $0.191 \pm 0.197$ \\
\hline & 0.0181 & 0.0145 & 0.0009 & 0.0339 & 0.0302 & 0.3344 \\
\hline \multicolumn{7}{|c|}{ Phosphatidylcholines ( $\mu \mathrm{M})$} \\
\hline \multirow[t]{2}{*}{ PC ae C32:2 } & $0.007 \pm 0.463$ & $0.029 \pm 0.087$ & $0.478 \pm 0.190$ & $0.114 \pm 0.271$ & $-0.026 \pm 0.012$ & $-0.229 \pm 0.177$ \\
\hline & 0.9886 & 0.7380 & 0.0133 & 0.6752 & 0.0390 & 0.2000 \\
\hline \multirow[t]{2}{*}{ PC ae C34:0 } & $-0.158 \pm 0.737$ & $0.284 \pm 0.138$ & $0.666 \pm 0.302$ & $0.075 \pm 0.431$ & $-0.041 \pm 0.020$ & $-0.080 \pm 0.282$ \\
\hline & 0.8312 & 0.0418 & 0.0295 & 0.8621 & 0.0441 & 0.7761 \\
\hline \multirow[t]{2}{*}{ PC ae C34:3 } & $15.585 \pm 3.858$ & $-1.788 \pm 0.721$ & $1.492 \pm 1.580$ & $-6.850 \pm 2.258$ & $-0.230 \pm 0.104$ & $-2.803 \pm 1.476$ \\
\hline & 0.0001 & 0.0148 & 0.3473 & 0.0031 & 0.0292 & 0.0605 \\
\hline \multirow[t]{2}{*}{ PC ae C36:2 } & $29.048 \pm 6.159$ & $-0.149 \pm 1.151$ & $-2.674 \pm 2.523$ & $-15.795 \pm 3.605$ & $-0.338 \pm 0.166$ & $-2.884 \pm 2.356$ \\
\hline & $<0.0001$ & 0.8969 & 0.2915 & $<0.0001$ & 0.0446 & 0.2238 \\
\hline \multirow[t]{2}{*}{ PC ae $C 40: 1$} & $2.153 \pm 0.872$ & $0.357 \pm 0.163$ & $0.862 \pm 0.357$ & $-0.748 \pm 0.510$ & $-0.074 \pm 0.024$ & $-0.253 \pm 0.334$ \\
\hline & 0.0152 & 0.0306 & 0.0176 & 0.1456 & 0.0020 & 0.4491 \\
\hline \multicolumn{7}{|l|}{ Amino acids $(\mu \mathrm{M})$} \\
\hline \multirow[t]{2}{*}{ Gln } & $865.339 \pm 430.321$ & $-124.218 \pm 80.390$ & $-303.854 \pm 176.254$ & $-428.871 \pm 251.864$ & $-32.233 \pm 11.602$ & $-127.624 \pm 164.646$ \\
\hline & 0.0469 & 0.1254 & 0.0877 & 0.0916 & 0.0065 & 0.4400 \\
\hline
\end{tabular}

Multiple linear regression analysis was used to perform the analysis after adjusting for age, sex and body mass index with total cholesterol, triglycerides, $\mathrm{HDL}-\mathrm{C}$, LDL-C, serum $\mathrm{Hp}$ level tertiles and carotid IMT as independent variables. $\beta \pm \mathrm{SE}$ and $P$ values are shown in table

Non-DM non-diabetes mellitus, Hp haptoglobin, Gln Glutamine, HDL-C high-density lipoprotein-cholesterol, LDL-C low-density lipoprotein-cholesterol, IMT intimamedia thickness

$P$ values $<0.05$ are shown in italic
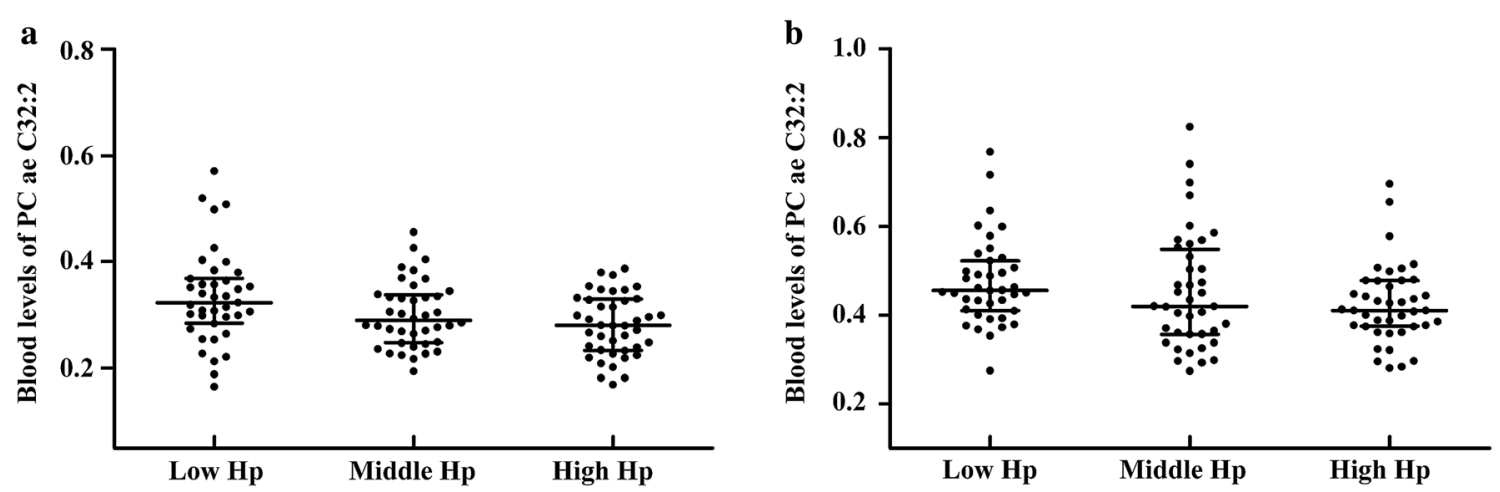

Fig. 2 Blood levels of metabolite PC ae C32:2 in subjects grouped by serum Hp levels. Hp haptoglobin, T2DM type 2 diabetes mellitus, Non-DM non-diabetes mellitus. Dot plot a shows the association of blood metabolite PC ae C32:2 with serum Hp tertiles in T2DM patients, $P=0.0005$, $\beta \pm S E=-0.028 \pm 0.008$. Dot plot $\mathbf{b}$ shows the association of blood metabolite $P C$ ae $C 32: 2$ with serum Hp tertiles in non-DM patients, $P=0.0390$ $\beta \pm S E=-0.026 \pm 0.012$. $P$ values and beta values were determined by multiple linear regression adjusting for age, sex, body mass index, total cholesterol, triglycerides, high-density lipoprotein-cholesterol, low-density lipoprotein-cholesterol, inter-adventitial diameter and/or carotid intima-media thickness. The PC ae C32:2 levels are shown in dot plots; the median is indicated by the middle black solid line. The lower and upper quartiles are shown by the bottom and top black solid lines, respectively 
Table 5 Metabolite ratios correlated with blood lipids and serum Hp levels in T2DM patients

\begin{tabular}{llllllll}
\hline Metabolite ratios & Total cholesterol & Triglycerides & HDL-C & LDL-C & Serum Hp & Carotid IMT & Carotid IAD \\
\hline Asn/PC ae C32:2 & $-0.949 \pm 0.290$ & $0.061 \pm 0.066$ & $-0.006 \pm 0.137$ & $0.277 \pm 0.164$ & $0.033 \pm 0.012$ & $0.344 \pm 0.114$ & $-0.230 \pm 0.196$ \\
& 0.0015 & 0.3555 & 0.9626 & 0.0942 & 0.0059 & 0.0032 & 0.2433 \\
Asp/PC ae C32:2 & $-0.723 \pm 0.367$ & $0.047 \pm 0.083$ & $-0.153 \pm 0.173$ & $0.290 \pm 0.207$ & $0.041 \pm 0.015$ & $0.361 \pm 0.144$ & $-0.516 \pm 0.248$ \\
& 0.0513 & 0.5726 & 0.3789 & 0.1651 & 0.0059 & 0.0138 & 0.0396 \\
Glu/PC ae C32:2 & $-1.121 \pm 0.400$ & $0.218 \pm 0.091$ & $-0.113 \pm 0.189$ & $0.554 \pm 0.226$ & $0.039 \pm 0.016$ & $0.252 \pm 0.157$ & $-0.817 \pm 0.270$ \\
& 0.0061 & 0.0183 & 0.5520 & 0.0160 & 0.0160 & 0.1125 & 0.0031 \\
Ile/PC ae C32:2 & $-1.084 \pm 0.329$ & $0.241 \pm 0.075$ & $-0.037 \pm 0.156$ & $0.473 \pm 0.186$ & $0.031 \pm 0.013$ & $0.170 \pm 0.129$ & $-0.534 \pm 0.222$ \\
& 0.0014 & 0.0017 & 0.8101 & 0.0124 & 0.0222 & 0.1933 & 0.0180 \\
Val/PC ae C32:2 & $-1.011 \pm 0.300$ & $0.189 \pm 0.068$ & $-0.071 \pm 0.142$ & $0.499 \pm 0.169$ & $0.031 \pm 0.012$ & $0.183 \pm 0.118$ & $-0.569 \pm 0.202$ \\
& 0.0010 & 0.0064 & 0.6197 & 0.0039 & 0.0116 & 0.1241 & 0.0059 \\
\hline
\end{tabular}

Multiple linear regression analysis was used to perform the analysis after adjusting for age, sex and body mass index with total cholesterol, triglycerides, $\mathrm{HDL}-\mathrm{C}$, $\mathrm{LDL}-\mathrm{C}$, serum $\mathrm{Hp}$ level tertiles, carotid IMT and IAD as independent variables. $\beta \pm \mathrm{SE}$ and $P$ values are shown in table

T2DM type 2 diabetes mellitus, Asn asparagine, Asp aspartate, Glu glutamic acid, Ile isoleucine, Val valine, Hp haptoglobin, HDL-C high-density lipoprotein-cholesterol, $L D L$-C low-density lipoprotein-cholesterol, IMT intima-media thickness, IAD inter-adventitial diameter

$P$ values $<0.05$ are shown in italic

Table 6 Metabolite ratios correlated with blood lipids and serum Hp levels in non-DM subjects

\begin{tabular}{lllllll}
\hline Metabolite ratios & Total cholesterol & Triglycerides & HDL-C & LDL-C & Serum Hp & Carotid IMT \\
\hline Asp/PC ae C32:2 & $0.028 \pm 0.538$ & $-0.072 \pm 0.101$ & $-0.605 \pm 0.220$ & $-0.254 \pm 0.315$ & $0.048 \pm 0.015$ & $0.362 \pm 0.206$ \\
& 0.9592 & 0.4748 & 0.0071 & 0.4212 & 0.0012 & 0.0820 \\
Glu/PC ae C32:2 & $-0.830 \pm 0.584$ & $0.020 \pm 0.109$ & $-0.442 \pm 0.239$ & $0.317 \pm 0.342$ & $0.043 \pm 0.016$ & $0.386 \pm 0.223$ \\
& 0.1585 & 0.8514 & 0.0673 & 0.3559 & 0.0071 & 0.0870 \\
Phe/PC ae C32:2 & $-0.311 \pm 0.407$ & $-0.052 \pm 0.076$ & $-0.493 \pm 0.167$ & $0.062 \pm 0.238$ & $0.029 \pm 0.011$ & $0.351 \pm 0.156$ \\
& 0.4470 & 0.4945 & 0.0039 & 0.7948 & 0.0094 & 0.0267 \\
\hline
\end{tabular}

Multiple linear regression analysis was used to perform the analysis after adjusting for age, sex and body mass index with total cholesterol, triglycerides, $\mathrm{HDL}-\mathrm{C}$, LDL-C, serum $\mathrm{Hp}$ level tertiles and carotid IMT as independent variables. $\beta \pm \mathrm{SE}$ and $P$ values are shown in table

Non-DM non-diabetes mellitus, Asp aspartate, Glu glutamic acid, Phe phenylalanine, Hp haptoglobin, HDL-C high-density lipoprotein-cholesterol, LDL-C low-density lipoprotein-cholesterol, IMT intima-media thickness

$P$ values $<0.05$ or approximately 0.05 are shown in italic
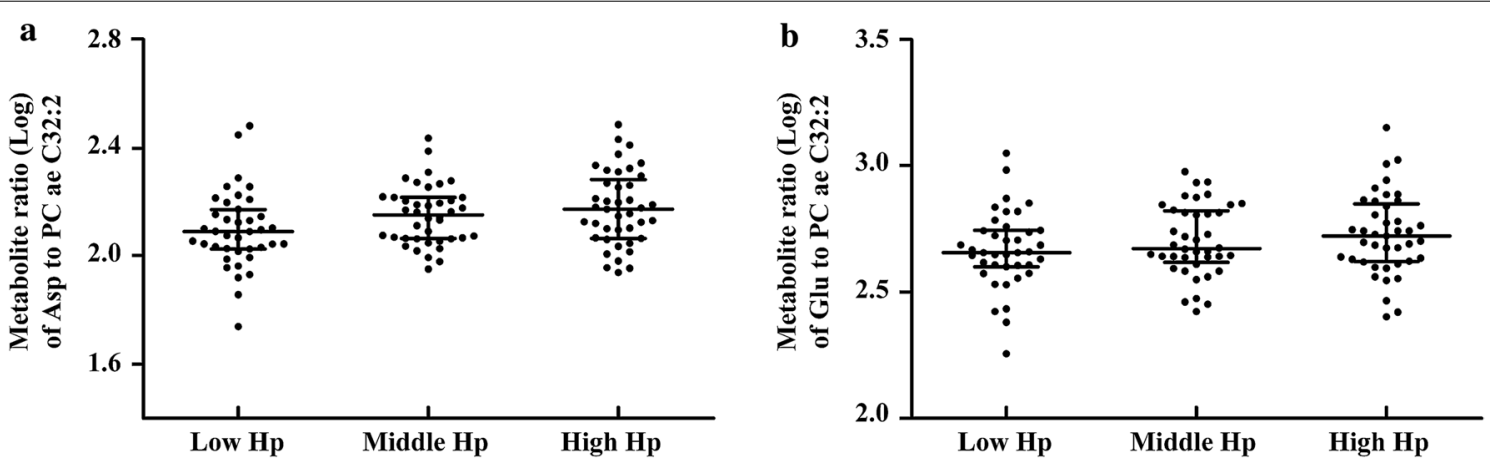

Fig. 3 Metabolite ratios of two acidic amino acids to PC ae C32:2 in T2DM patients. T2DM type 2 diabetes mellitus, Hp haptoglobin, Asp aspartate, Glu glutamate. Dot plot a shows the association of blood metabolite ratio Asp to PC ae C32:2 (Log transformed) with serum Hp tertiles, $P=0.0059$, $\beta \pm S E=0.041 \pm 0.015$. Dot plot $\mathbf{b}$ shows the association of blood metabolite ratio Glu to PC ae C32:2 (Log transformed) with serum Hp tertiles, $P=0.0160, \beta \pm \mathrm{SE}=0.039 \pm 0.016$. $P$ values and beta values were determined by multiple linear regression adjusting for age, sex, body mass index, total cholesterol, triglycerides, high-density lipoprotein-cholesterol, low-density lipoprotein-cholesterol, carotid intima-media thickness and inter-adventitial diameter. Metabolite ratios (Log transformed) are shown in dot plots; the median is indicated by the middle black solid line. The lower and upper quartiles are shown by the bottom and top black solid lines, respectively 

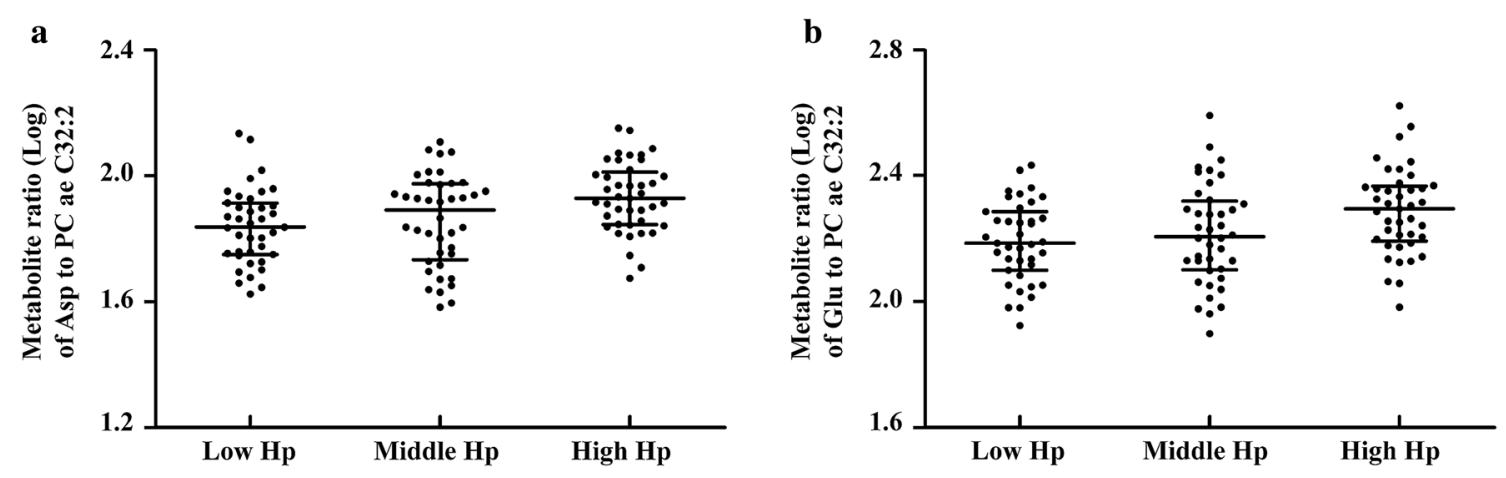

Fig. 4 Metabolite ratios of two acidic amino acids to PC ae C32:2 in non-DM subjects. Non-DM non-diabetes mellitus, Hp haptoglobin, Asp aspartate, Glu glutamate. Dot plot a shows the association of blood metabolite ratio Asp to PC ae C32:2 (Log transformed) with serum Hp tertiles, $P=0.0012, \beta \pm S E=0.048 \pm 0.015$. Dot plot $\mathbf{b}$ shows the association of blood metabolite ratio Glu to PC ae C32:2 (Log transformed) with serum Hp tertiles, $P=0.0071, \beta \pm \mathrm{SE}=0.043 \pm 0.016$. $P$ values and beta values were determined by multiple linear regression adjusting for age, sex, body mass index, total cholesterol, triglycerides, high-density lipoprotein-cholesterol, low-density lipoprotein-cholesterol and carotid intima-media thickness. Metabolite ratios (Log transformed) are shown in dot plots; the median is indicated by the middle black solid line. The lower and upper quartiles are shown by the bottom and top black solid lines, respectively

PCs catalysed mainly by phospholipase A1 (PLA1) and phospholipase A2 (PLA2), lecithin-cholesterol acyltransferase (LCAT) activity or hepatic secretion [31]. Due to the diverse composition of fatty acid residues binding to the glycerol backbone, individual PCs and lyso-PCs have different functional properties and reactions to oxidative stress [32-35]. A reduction in the levels of several individual PC and lyso-PC species has previously been reported in patients with atherosclerosis in comparison to those of healthy subjects via a targeted metabolomics approach [36], indicating that abnormal glycerophospholipid metabolism might be correlated with the aberrant activity of several enzymes, such as PLA1, PLA2 and LCAT, crucial components of oxidized LDL (Ox-LDL), which contributes to atherogenesis $[37,38]$.

In our study, the levels of 13 glycerophospholipids (1 lyso-PC and $12 \mathrm{PCs}$ ) and 9 glycerophospholipids (2 lyso-PC and 7 PCs) were observed to be significantly lower in the upper serum $\mathrm{Hp}$ tertile group than in the lower tertile group in T2DM patients and non-DM subjects, respectively. As reported previously, it was speculated that the influence of $\mathrm{Hp}$ on the cholesterol level might be related to the ability of $\mathrm{Hp}$ to bind apolipoprotein $\mathrm{E}$, which results in the displacement and inhibition of the LCAT cholesterol esterification rate [18]. The decrease in levels of PC and lyso-PC species in patients with higher serum $\mathrm{Hp}$ concentrations, which might be caused by aberrant enzyme activity, provide a likely explanation for the potential mechanism underlying the interaction of serum $\mathrm{Hp}$ and lipid metabolism.

One highlight of the glycerophospholipids associated with serum $\mathrm{Hp}$ is PC ae C32:2, which was shown to be significantly correlated with carotid IAD in T2DM patients. Carotid IAD and IMT measured by ultrasound serve as a non-invasive surrogate for assessing the atherosclerotic process $[39,40]$. Although carotid IMT is recommended for early detection and cardiovascular risk evaluation, the role of carotid IAD in cardiovascular outcomes prediction has gradually been recognized [41, 42]. Several studies observed that common carotid IAD and not carotid IMT was correlated with the prevalence of cardiovascular events and might have predictive value for cardiovascular outcomes [43, 44]. In our research, metabolite PC ae C32:2 was correlated with serum $\mathrm{Hp}$ and common carotid IAD in T2DM patients with a median diabetes duration of 10 years. We speculated that the lack of associations between metabolites and carotid IMT might due to several factors. One factor to take into consideration is vascular remodelling. With the deposition and development of atherosclerotic plaques, vascular remodelling occurs and results in increased carotid diameter, called the Glagov phenomenon [45]. As a sum of the lumen diameter and of IMT, carotid IAD is associated with carotid IMT, left ventricular mass and cardiovascular risk factors, which is more obvious to reflect the change of carotid arterial functions [46]. The other is the possibility that carotid IMT is more sensitive to metabolites in those patients with mild impaired glucose regulation or lower burdens of subclinical cardiovascular diseases than those with larger burden T2DM patients. Further studies should be carried out to cast insights into the mechanism behind the association of serum $\mathrm{Hp}$ and carotid arterial functions.

Interestingly, changes in the ratios between two single metabolites might reflect biological situations such as 
alterations in enzyme activities or imbalances in metabolic pathways relevant for a certain phenotype [47-49]. A study reported recently that the metabolite ratio of $\mathrm{Val} / \mathrm{PC}$ ae C32:2 was not only associated with measures of oral glucose tolerance test -derived $\beta$ cell function and insulin resistance but also correlated with an increased risk of T2DM [27]. The observed effects, independent of currently known risk factors, were stronger than those of the individual metabolites, indicating that the application of ratios might improve prediction above that of single metabolites.

Based on this background, the metabolite ratios of 21 amino acids to PC ae C32:2 were compared in this study to evaluate the association with serum $\mathrm{Hp}$ levels and clinical traits. As a result, we identified that the metabolite ratios of two acidic amino acids, including Asp/PC ae C32:2 and Glu/PC ae C32:2 were significantly correlated with serum Hp levels, carotid IMT or IAD, and other lipid metabolic parameters after adjusting for confounding factors. As reported previously, acidic amino acids, including aspartate and glutamate were identified to have an intrinsic peroxidase-like activity, which plays a key role in lipid oxidation [50]. It was speculated that metabolites found in our study might mediate the aberrant activity of several enzymes caused by the action of $\mathrm{Hp}$ binding to apolipoprotein $\mathrm{E}$ and apolipoprotein A-I, which disturbs cholesterol esterification and lipid oxidation, contributing to blood vessel endothelial dysfunction and atherogenesis $[37,38]$. Changes in those metabolite ratios might reflect the alterations in enzyme activities relevant to the rate-limiting step in glycolipid catabolism $[51,52]$. In view of this, we speculated that it might be useful for early identification of subjects with an increased risk of cardio-cerebrovascular diseases or helpful to identify potential drug targets for new therapies if verified by further prospective studies [53-55].

To the best of our knowledge, this is the first study to use a targeted metabolomics approach to analyse blood metabolites in relation to serum Hp levels and carotid arterial functions. However, there are several limitations in this research. First, the sample we recruited to perform metabolite measurements was relatively small, and large-scale studies are needed to confirm our results. Second, part of the explanation for our results is limited by the targeted metabolomics kit we used, as it does not provide a detailed analysis of the lipid composition of metabolites, such as PC ae C32:2. Its exact role in the physiology and molecular aetiology awaits examination in further functional studies. Third, as carotid IMT is recommended for early detection and cardiovascular risk evaluation in general population, carotid IAD was not recorded in non-DM subjects in this study. The association between serum Hp levels and carotid arterial functions should be evaluated in other complex diseases. Finally, a direct causal relationship cannot be established in view of the cross-sectional design of this study. Therefore, the findings identified in our study should be further validated in a prospective study.

\section{Conclusions}

To summarize, we show that a number of blood metabolites, especially PCs and lyso-PCs, were associated with serum Hp levels and blood lipid metabolism by using a targeted metabolomics approach in Chinese T2DM patients and non-DM subjects. This finding provides a new insight into potential mechanisms behind the interaction between serum Hp and carotid arterial functions. Furthermore, the metabolite ratios of two acidic amino acids, including Asp/PC ae C32:2 and Glu/PC ae C32:2 may be used to predict early risk of cardio-cerebrovascular diseases if validated in further studies.

\section{Additional file}

Additional file 1: Table S1. Correlation between clinical traits and serum $\mathrm{Hp}$ levels in T2DM patients. Table S2. Correlation between clinical traits and serum Hp levels in non-DM subjects. Table S3. Blood metabolites associated with serum Hp levels. Table S4. Metabolite ratios correlated with clinical traits in T2DM patients. Table S5. Metabolite ratios correlated with clinical traits in non-DM subjects. Figure S1. Serum Hp levels in nondiabetes mellitus subjects.

\section{Abbreviations}

T2DM: type 2 diabetes mellitus; Non-DM: non-diabetes mellitus; Hp: haptoglobin; IMT: intima-media thickness; IAD: inter-adventitial diameter; BMI: body mass index; HbA1c: haemoglobin A1c; HDL-C: high-density lipoproteincholesterol; LDL-C: low-density lipoprotein-cholesterol; lyso-PCs: lyso-phosphatidylcholines; PCs: phosphatidylcholines; UPLC: ultra performance liquid chromatography; $\mu \mathrm{M}$ : $\mu \mathrm{mol} / \mathrm{L} ;$ ELISA: enzyme-linked immunosorbent assay; ANOVA: analysis of variance; $\mathrm{Hb}-\mathrm{Hp}$ : haemoglobin-haptoglobin; PLA1: phospholipase A1; PLA2: phospholipase A2; LCAT: lecithin-cholesterol acyltransferase; Ox-LDL: oxidized LDL.

\section{Authors' contributions}

SW and JW contributed equally to this work. CH and WJ conceived of and designed the research. JW, WJ, FJ and DY contributed reagents/materials and tools. SW, JW, RZ, AZ and XZ performed the experiments and analysed the data. SW and JW drafted the manuscript. All authors contributed to the writing of the manuscript. All authors read and approved the final manuscript.

\footnotetext{
Author details

${ }^{1}$ Shanghai Diabetes Institute, Shanghai Key Laboratory of Diabetes Mellitus, Shanghai Clinical Center for Diabetes, Shanghai Jiao Tong University Affiliated Sixth People's Hospital, 600 Yishan Road, Shanghai 200233, People's Republic of China. ${ }^{2}$ Center for Translational Medicine, Shanghai Key Laboratory of Diabetes Mellitus, Department of Endocrinology and Metabolism, Shanghai Jiao Tong University Affiliated Sixth People's Hospital, 600 Yishan Road, Shanghai 200233, People's Republic of China. ${ }^{3}$ Institute for Metabolic Disease, Fengxian Central Hospital Affiliated to Southern Medical University, 6600 Nanfeng Road, Shanghai 201499, People's Republic of China.
} 


\section{Acknowledgements}

We thank all the research subjects for their participation and acknowledge the supports of Biobank of Shanghai Jiao Tong University Affiliated Sixth People's Hospital, the skilful work of the entire medical staff at the Shanghai Clinical Center for Diabetes, Center for Translational Medicine and Shanghai Key Laboratory of Diabetes Mellitus.

\section{Competing interests}

The authors declare that they have no competing interests.

\section{Availability of data and materials}

All data generated or analysed during this study are included in this published article and its additional files.

\section{Consent for publication}

Not applicable.

\section{Ethics approval and consent to participate}

Ethical approval was granted by the Institutional Review Board of Shangha Jiao Tong University Affiliated Sixth People's Hospital according to Helsinki Declaration II. All subjects provided written informed consent to participate.

\section{Funding}

This work was financially supported by grants from the National Key Research and Development Project of China (2016YFC0903303); National Natural Science Foundation of China grants (81570713 and 91649112); Outstanding Academic Leaders of Shanghai Health System (2017BR008); the National Program for Support of Top-notch Young Professionals; and Yangtze River Scholar.

\section{Publisher's Note}

Springer Nature remains neutral with regard to jurisdictional claims in published maps and institutional affiliations.

Received: 23 August 2018 Accepted: 3 January 2019

Published online: 11 January 2019

\section{References}

1. Murray CJ, Vos T, Lozano R, Naghavi M, Flaxman AD, Michaud C, Ezzati M, Shibuya K, Salomon JA, Abdalla S, et al. Disability-adjusted life years (DALYS) for 291 diseases and injuries in 21 regions, 1990-2010: a systematic analysis for the Global Burden of Disease Study 2010. Lancet. 2012;380(9859):2197-223.

2. Gu K, Cowie CC, Harris MI. Mortality in adults with and without diabetes in a national cohort of the U.S. population, 1971-1993. Diabetes Care. 1998;21(7):1138-45.

3. Newman JD, Schwartzbard AZ, Weintraub HS, Goldberg IJ, Berger JS. Primary prevention of cardiovascular disease in diabetes mellitus. J Am Coll Cardiol. 2017;70(7):883-93.

4. Mellbin LG, Anselmino M, Ryden L. Diabetes, prediabetes and cardiovascular risk. Eur J Cardiovasc Prev Rehabil. 2010;17(Suppl 1):S9-14.

5. Bao X, Borne Y, Johnson L, Muhammad IF, Persson M, Niu K, Engstrom G. Comparing the inflammatory profiles for incidence of diabetes mellitus and cardiovascular diseases: a prospective study exploring the 'common soil' hypothesis. Cardiovasc Diabetol. 2018;17(1):87.

6. Ijas P, Saksi J, Soinne L, Tuimala J, Jauhiainen M, Jula A, Kahonen M, Kesaniemi YA, Kovanen PT, Kaste M, et al. Haptoglobin 2 allele associates with unstable carotid plaque and major cardiovascular events. Atherosclerosis. 2013;230(2):228-34

7. Dalan R, Liew H, Goh LL, Gao X, Chew DE, Boehm BO, Leow MK. The haptoglobin 2-2 genotype is associated with inflammation and carotid artery intima-media thickness. Diabetes Vasc Dis Res. 2016;13(5):373-6.

8. Orchard TJ, Backlund JC, Costacou T, Cleary P, Lopes-Virella M, Levy AP, Lachin JM, Group DER. Haptoglobin 2-2 genotype and the risk of coronary artery disease in the Diabetes Control and Complications Trial/ Epidemiology of Diabetes Interventions and Complications study (DCCT/ EDIC). J Diabetes Complications. 2016:30(8):1577-84.

9. Jansen H, Samani NJ, Schunkert H. Mendelian randomization studies in coronary artery disease. Eur Heart J. 2014;35(29):1917-24.
10. Jimenez-Rosales A, Amaya-Chavez A, Dominguez Garcia MV, CamarilloRomero E, Huitron Bravo GG, Cruz AM. Association of inflammatory and oxidative stress biomarkers in subjects with cardiovascular risk. Am J Ther. 2013;20(4):422-31.

11. Xuan Y, Gao X, Holleczek B, Brenner H, Schottker B. Prediction of myocardial infarction, stroke and cardiovascular mortality with urinary biomarkers of oxidative stress: results from a large cohort study. Int J Cardiol. 2018;273:223-9. https://doi.org/10.1016/j.ijcard.2018.08.002

12. Wang S, Wang J, Zhang R, Wang T, Yan D, He Z, Jiang F, Hu C, Jia W. Mendelian randomization analysis to assess a causal effect of haptoglobin on macroangiopathy in Chinese type 2 diabetes patients. Cardiovasc Diabetol. 2018;17(1):14.

13. Andersen CBF, Stodkilde K, Saederup KL, Kuhlee A, Raunser S, Graversen JH, Moestrup SK. Haptoglobin. Antioxid Redox Signal. 2017;26(14):814-31.

14. Alayash Al, Andersen CB, Moestrup SK, Bulow L. Haptoglobin: the hemoglobin detoxifier in plasma. Trends Biotechnol. 2013;31(1):2-3.

15. Can U, Buyukinan M, Guzelant A, Ugur A, Karaibrahimoglu A, Yabanciun S. Investigation of the inflammatory biomarkers of metabolic syndrome in adolescents. J Pediatr Endocrinol Metab. 2016;29(11):1277-83.

16. Awadallah S, Madkour M, Hamidi RA, Alwafa EA, Hattab M, Zakkour B, Al-Matroushi A, Ahmed E, Al-Kitbi M. Plasma levels of apolipoprotein A1 and lecithin: cholesterol acyltransferase in type 2 diabetes mellitus: correlations with haptoglobin phenotypes. Diabetes Metab Syndr. 2017;11(Suppl 2):S543-6.

17. Melander O, Modrego J, Zamorano-Leon JJ, Santos-Sancho JM, Lahera V, Lopez-Farre AJ. New circulating biomarkers for predicting cardiovascular death in healthy population. J Cell Mol Med. 2015:19(10):2489-99.

18. Cigliano L, Pugliese CR, Spagnuolo MS, Palumbo R, Abrescia P. Haptoglobin binds the antiatherogenic protein apolipoprotein E-impairment of apolipoprotein E stimulation of both lecithin:cholesterol acyltransferase activity and cholesterol uptake by hepatocytes. FEBS J. 2009;276(21):6158-71.

19. Sas KM, Karnovsky A, Michailidis G, Pennathur S. Metabolomics and diabetes: analytical and computational approaches. Diabetes. 2015;64(3):718-32.

20. Newgard CB. Metabolomics and metabolic diseases: where do we stand? Cell Metab. 2017;25(1):43-56.

21. Johnson CH, Ivanisevic J, Siuzdak G. Metabolomics: beyond biomarkers and towards mechanisms. Nat Rev Mol Cell Biol. 2016;17(7):451-9.

22. Lu J, Xie G, Jia W, Jia W. Metabolomics in human type 2 diabetes research. Front Med. 2013;7(1):4-13.

23. Klupczynska A, Derezinski P, Kokot ZJ. Metabolomics in medical sciencestrends, challenges and perspectives. Acta Pol Pharm. 2015;72(4):629-41.

24. Chen P, Hou X, Hu G, Wei L, Jiao L, Wang H, Chen S, Wu J, Bao Y, Jia W. Abdominal subcutaneous adipose tissue: a favorable adipose depot for diabetes? Cardiovasc Diabetol. 2018;17(1):93.

25. Qiu Y, Zhou B, Su M, Baxter S, Zheng X, Zhao X, Yen Y, Jia W. Mass spectrometry-based quantitative metabolomics revealed a distinct lipid profile in breast cancer patients. Int J Mol Sci. 2013;14(4):8047-61.

26. Suhre K, Raffler J, Kastenmuller G. Biochemical insights from population studies with genetics and metabolomics. Arch Biochem Biophys. 2016;589:168-76

27. Molnos S, Wahl S, Haid M, Eekhoff EMW, Pool R, Floegel A, Deelen J, Much D, Prehn C, Breier M, et al. Metabolite ratios as potential biomarkers for type 2 diabetes: a DIRECT study. Diabetologia. 2018;61(1):117-29.

28. Stegemann C, Pechlaner R, Willeit P, Langley SR, Mangino M, Mayr U, Menni C, Moayyeri A, Santer P, Rungger G, et al. Lipidomics profiling and risk of cardiovascular disease in the prospective population-based Bruneck study. Circulation. 2014;129(18):1821-31.

29. Wang Z, Klipfell E, Bennett BJ, Koeth R, Levison BS, Dugar B, Feldstein AE, Britt EB, Fu X, Chung YM, et al. Gut flora metabolism of phosphatidylcholine promotes cardiovascular disease. Nature. 2011:472(7341):57-63.

30. Glomset JA. The mechanism of the plasma cholesterol esterification reaction: plasma fatty acid transferase. Biochim Biophys Acta. 1962;65:128-35.

31. Chen L, Liang B, Froese DE, Liu S, Wong JT, Tran K, Hatch GM, Mymin D, Kroeger EA, Man RY, et al. Oxidative modification of low density lipoprotein in normal and hyperlipidemic patients: effect of lysophosphatidylcholine composition on vascular relaxation. J Lipid Res. 1997;38(3):546-53.

32. Oka H, Kugiyama K, Doi H, Matsumura T, Shibata H, Miles LA, Sugiyama S, Yasue $\mathrm{H}$. Lysophosphatidylcholine induces urokinase-type plasminogen 
activator and its receptor in human macrophages partly through redoxsensitive pathway. Arterioscler Thromb Vasc Biol. 2000;20(1):244-50

33. Kim M, Jung S, Kim SY, Lee SH, Lee JH. Prehypertension-associated elevation in circulating lysophosphatidlycholines, Lp-PLA2 activity, and oxidative stress. PLoS ONE. 2014;9(5):e96735.

34. Li X, Fang P, Li Y, Kuo YM, Andrews AJ, Nanayakkara G, Johnson C, Fu $H$, Shan H, Du F, et al. Mitochondrial reactive oxygen species mediate lysophosphatidylcholine-induced endothelial cell activation. Arterioscler Thromb Vasc Biol. 2016;36(6):1090-100.

35. Takahashi M, Okazaki H, Ogata Y, Takeuchi K, Ikeda U, Shimada K. Lysophosphatidylcholine induces apoptosis in human endothelial cells through a p38-mitogen-activated protein kinase-dependent mechanism. Atherosclerosis. 2002:161(2):387-94.

36. Paapstel K, Kals J, Eha J, Tootsi K, Ottas A, Piir A, Jakobson M, Lieberg J, Zilmer M. Inverse relations of serum phosphatidylcholines and lysophosphatidylcholines with vascular damage and heart rate in patients with atherosclerosis. Nutr Metab Cardiovasc Dis. 2018;28(1):44-52.

37. Mannheim D, Herrmann J, Versari D, Gossl M, Meyer FB, McConnell JP, Lerman LO, Lerman A. Enhanced expression of Lp-PLA2 and lysophosphatidylcholine in symptomatic carotid atherosclerotic plaques. Stroke. 2008;39(5):1448-55.

38. Nakhjavani M, Asgharani F, Khalilzadeh O, Esteghamati A, Ghaneei A, Morteza A, Anvari M. Oxidized low-density lipoprotein is negatively correlated with lecithin-cholesterol acyltransferase activity in type 2 diabetes mellitus. Am J Med Sci. 2011;341(2):92-5.

39. Eigenbrodt ML, Bursac Z, Rose KM, Couper DJ, Tracy RE, Evans GW, Brancati FL, Mehta JL. Common carotid arterial interadventitial distance (diameter) as an indicator of the damaging effects of age and atherosclerosis, a cross-sectional study of the Atherosclerosis Risk in Community Cohort Limited Access Data (ARICLAD), 1987-89. Cardiovasc Ultrasound. 2006:4:1.

40. Naqvi TZ, Lee MS. Carotid intima-media thickness and plaque in cardiovascular risk assessment. JACC Cardiovasc Imaging. 2014;7(10):1025-38

41. Saba L, Araki T, Kumar PK, Rajan J, Lavra F, Ikeda N, Sharma AM, Shafique S, Nicolaides A, Laird JR, et al. Carotid inter-adventitial diameter is more strongly related to plaque score than lumen diameter: an automated tool for stroke analysis. J Clin Ultrasound. 2016;44(4):210-20

42. Kozakova M, Morizzo C, La Carrubba S, Fabiani I, Della Latta D, Jamagidze J, Chiappino D, Di Bello V, Palombo C. Associations between common carotid artery diameter, Framingham risk score and cardiovascular events. Nutr Metab Cardiovasc Dis. 2017;27(4):329-34.

43. Polak JF, Sacco RL, Post WS, Vaidya D, Arnan MK, O'Leary DH. Incident stroke is associated with common carotid artery diameter and not common carotid artery intima-media thickness. Stroke. 2014;45(5):1442-6.

44. Eigenbrodt ML, Sukhija R, Rose KM, Tracy RE, Couper DJ, Evans GW, Bursac Z, Mehta JL. Common carotid artery wall thickness and external diameter as predictors of prevalent and incident cardiac events in a large population study. Cardiovasc Ultrasound. 2007;5:11
45. Glagov S, Weisenberg E, Zarins CK, Stankunavicius R, Kolettis GJ. Compensatory enlargement of human atherosclerotic coronary arteries. N Engl J Med. 1987:316(22):1371-5.

46. Polak JF, Wong Q, Johnson WC, Bluemke DA, Harrington A, O'Leary DH, Yanez ND. Associations of cardiovascular risk factors, carotid intima-media thickness and left ventricular mass with inter-adventitial diameters of the common carotid artery: the multi-ethnic study of atherosclerosis (MESA). Atherosclerosis. 2011;218(2):344-9.

47. Petersen AK, Krumsiek J, Wagele B, Theis FJ, Wichmann HE, Gieger C, Suhre K. On the hypothesis-free testing of metabolite ratios in genomewide and metabolome-wide association studies. BMC Bioinform. 2012;13:120.

48. Zhai G, Wang-Sattler R, Hart DJ, Arden NK, Hakim AJ, Illig T, Spector TD. Serum branched-chain amino acid to histidine ratio: a novel metabolomic biomarker of knee osteoarthritis. Ann Rheum Dis. 2010;69(6):1227-31.

49. Illig T, Gieger C, Zhai G, Romisch-MargI W, Wang-Sattler R, Prehn C, Altmaier E, Kastenmuller G, Kato BS, Mewes HW, et al. A genome-wide perspective of genetic variation in human metabolism. Nat Genet. 2010:42(2):137-41.

50. Shi Y, Liu L, Yu Y, Long Y, Zheng H. Acidic amino acids: a new-type of enzyme mimics with application to biosensing and evaluating of antioxidant behaviour. Spectrochim Acta A Mol Biomol Spectrosc 2018;201:367-75

51. Aftring RP, Manos PN, Buse MG. Catabolism of branched-chain amino acids by diaphragm muscles of fasted and diabetic rats. Metabolism. 1985;34(8):702-11.

52. Adeva-Andany MM, Lopez-Maside L, Donapetry-Garcia C, FernandezFernandez C, Sixto-Leal C. Enzymes involved in branched-chain amino acid metabolism in humans. Amino Acids. 2017:49(6):1005-28.

53. Katakami N, Mita T, Irie Y, Takahara M, Matsuoka TA, Gosho M, Watada $\mathrm{H}$, Shimomura I, Sitagliptin Preventive study of Intima-media thickness Evaluation C. Effect of sitagliptin on tissue characteristics of the carotid wall in patients with type 2 diabetes: a post hoc sub-analysis of the sitagliptin preventive study of intima-media thickness evaluation (SPIKE). Cardiovasc Diabetol. 2018;17(1):24.

54. Bhanpuri NH, Hallberg SJ, Williams PT, McKenzie AL, Ballard KD, Campbell WW, McCarter JP, Phinney SD, Volek JS. Cardiovascular disease risk factor responses to a type 2 diabetes care model including nutritional ketosis induced by sustained carbohydrate restriction at 1 year: an open label, non-randomized, controlled study. Cardiovasc Diabetol. 2018;17(1):56.

55. Rizzo M, Rizvi AA, Patti AM, Nikolic D, Giglio RV, Castellino G, Li Volti G, Caprio M, Montalto G, Provenzano V, et al. Liraglutide improves metabolic parameters and carotid intima-media thickness in diabetic patients with the metabolic syndrome: an 18-month prospective study. Cardiovasc Diabetol. 2016;15(1):162.

Ready to submit your research? Choose BMC and benefit from

- fast, convenient online submission

- thorough peer review by experienced researchers in your field

- rapid publication on acceptance

- support for research data, including large and complex data types

- gold Open Access which fosters wider collaboration and increased citations

- maximum visibility for your research: over $100 \mathrm{M}$ website views per year

At $\mathrm{BMC}$, research is always in progress.

Learn more biomedcentral.com/submissions 